\title{
Estudio in vitro de la relación entre resistencia de unión a esmalte dental y microfiltración en resinas compuestas fotopolimerizables
}

\author{
In vitro study of the relationship between bond strength to dental \\ enamel and microleakage in photopolymerizable composite resins \\ Estudio in vitro de la relación entre resistencia de unión a esmalte \\ dental y microfiltración en resinas compuestas fotopolimerizables
}

\author{
Maria Elisa de León Cáceres ORCID: 0000-0002-3780-1034 \\ Matias Mederos Gómez ${ }^{1}$ ORCID: 0000-0002-1561-2283 \\ Carlos Enrique Cuevas-Suárez² ORCID: 0000-0002-2759-8984 \\ Francisco Maglione García ${ }^{3}$ ORCID: 0000-0002-9491-1395 \\ Guillermo Steven Grazioli Pita' ORCID: 0000-0001-9969-3780
}

\section{Resumen}

Objetivos. Evaluar y comparar la relación entre la resistencia de unión inmediata a esmalte y microfiltración de dos sistemas restauradores a base de resina compuesta. Métodos. 40 terceros molares se dividieron aleatoriamente en dos grupos: 20 molares restaurados utilizando el sistema Adper Single bond 2 + Filtek Z250 XT (3M ESPE; ST PAUL, MN, USA); y 20 molares restaurados con el sistema Tetric N Bond + Tetric N Ceram (Ivoclar Vivadent; Schaan, Liechtenstein). La mitad de los dientes de cada grupo se utilizaron para un ensayo de microtracción, mientras que la otra mitad fueron utilizados para un ensayo de microfiltración $(\mathrm{n}=10)$.

Resultados. Las medias y el desvío padrón de la resistencia de unión a microtracción fueron de 27.93 ( \pm 9.55$)$ para Adper Single bond 2 + Filtek Z250 XT MPa y $33.12( \pm 8.18) \mathrm{MPa}$ para Tetric N Bond + Tetric N Ceram $(p=0,049)$. En cuanto a los valores de microfiltración, no hubo diferencias estadísticamente significativas entre los grupos $(p=0,478)$. No fue observada una correlación significativa entre la resistencia de unión a la microtracción y la microfiltración $\left(\mathrm{R}^{2}=0.0909 ; \mathrm{p}=0.196\right)$.

Conclusiones. No se encontró una relación entre los valores de resistencia de unión y el grado de microfiltración.

Palabras clave: microtracción, microfiltración, resinas compuestas.

Fecha de recibido: 13/04/2019 - Fecha de aceptado: 28/01/2020 


\section{Abstract}

Objectives. To evaluate and compare the relationship between enamel bond strength and microleakage of two composite based restorative systems.

Methods. 40 third molars were randomly divided into two groups: 20 molars restored with the Adper Single bond 2 + Filtek Z250 XT (3M ESPE; ST PAUL, MN, USA) system and 20 with the Tetric N Bond + Tetric N Ceram (Ivoclar Vivadent; Schaan, Liechtenstein) system. "Half of the teeth" in each group were subjected to a bond strength test, while the others were subjected to the microleakage test.

Results. The means and standard deviation of the bond strength resistance were 27.93 $( \pm 9.55) \mathrm{MPa}$ for Adper Single bond $2+$ Filtek Z250 XT system and $33.12( \pm 8.18)$ $\mathrm{MPa}$ for Tetric $\mathrm{N}$ Bond + Tetric N Ceram system $(\mathrm{p}=0.049)$. Regarding microleakage values, there were no statistically significant differences between the groups $(\mathrm{p}=0.478)$. No significant correlation was observed between microtensile bond strength and microleakage $\left(\mathrm{R}^{2}=0.0909\right.$, $\mathrm{p}=0.196)$.

Conclusions. No relationship was found between adhesive bond values and degree of microleakage.

Keywords: bond strength, microleakage, composite resins.

\section{Introducción}

Desde su introducción en los años 60s, las resinas compuestas (RC) se han vuelto cada vez más utilizadas. En la actualidad, se consideran materiales de uso universal, siendo la primera opción para restauraciones directas en dientes anteriores y posteriores ${ }^{(1)}$. Las características estéticas que brindan al igualar el color natu-

\section{Resumo}

Objetivos. Avaliar e comparar a relação entre a resistência de união imediata ao esmalte e a microfiltração de dois sistemas restauradores baseados em resina composta.

Métodos. 40 terceiros molares foram divididos aleatoriamente em dois grupos: 20 molares restaurados usando o sistema Adper Single bond 2 + Filtek Z250 XT (3M ESPE; ST PAUL, MN, EUA); e 20 molares restaurados com o sistema Tetric N Bond + Tetric N Ceram (Ivoclar Vivadent; Schaan, Liechtenstein). Metade dos dentes de cada grupo foi utilizada para um teste de resistência de união à microtração, enquanto a outra metade foi utilizada para um teste de microfiltração $(\mathrm{n}=10)$.

Resultados. As mídias e o padrão de resistência de união à microtração foram 27,93 $( \pm 9,55)$ para Adper Single bond $2+$ Filtek Z250 XT MPa e 33,12 $( \pm 8,18) \mathrm{MPa}$ para Tetric N Bond + Tetric N Ceram $(\mathrm{p}=$ 0,049). Em relação aos valores de microfiltração, não houve diferença estatisticamente significativas entre os grupos $(p=0,478)$. Não houve correlação significativa observada entre a resistência de união à microtração e a microfiltração $\left(R^{2}=0,0909 ; \mathrm{p}=0,196\right)$. Conclusóes. Não foi encontrada relação entre os valores de resistência de união e o grau de microfiltração.

Palavras-chave: microtração; microfiltração; resinas compostas.

ral del diente, su capacidad de adherirse a los tejidos duros dentarios a través de sistemas adhesivos de micro-retención y el bajo costo en comparación con los materiales de restauración indirectos son algunas de las razones que justifican su uso ${ }^{(2)}$.

Sin embargo, aún se reportan gran cantidad de casos en los que este tipo de restauraciones fallan a corto y mediano plazo $^{(2)}$. Esto es debido 
a que existen problemas relacionados con los cambios que sufre la resina una vez polimerizada (contracción de polimerización, coeficiente de expansión térmica), y en relación con el ambiente bucal (abrasión superficial, degradación química, etc. $)^{(3,4)}$. Estos problemas son responsables del fracaso de las restauraciones, produciendo fallas en la interfaz diente-restauración, generando microfiltración y su consecuente recidiva de caries ${ }^{(5-8)}$

Para contrarrestar estos problemas, los fabricantes han realizado diferentes modificaciones en su composición, por ejemplo, monómeros con mayor peso molecular (produciendo menor contracción de polimerización), o bien introduciendo mejoras en las partículas de carga inorgánicas (mayor carga, menor tamaño y morfologías distintas), lo cual condujo a mejoras significativas en las propiedades del material $^{(9,10)}$.

Para el estudio del comportamiento de materiales restauradores a largo plazo, el uso de técnicas de envejecimiento acelerado en condiciones estandarizadas de laboratorio son una alternativa efectiva, ya que permiten la simulación del uso clínico de un material en un período corto de tiempo. Para este fin, algunos métodos han sido propuestos, entre los que se encuentran la inmersión en agua, etanol o saliva artificial, así como el ciclado térmico y el envejecimiento por radiación UV ${ }^{(11)}$.

El termociclado es el proceso de someter las muestras a temperaturas extremas para simular las condiciones cambiantes de temperatura intraoral $^{(12)}$. El efecto del termociclado induce la degradación de la interfaz diente/restauración debido a la diferencia en su coeficiente de expansión térmica ${ }^{(13)}$. Esta metodología es propuesta por la organización internacional de estandarización en su normativa ISO 11405 como un método para acelerar el envejecimiento de los materiales, simulando su sobrevida en boca $^{(14)}$.

Es bien conocida la simpleza y efectividad del proceso de adhesión a esmalte ${ }^{(15)}$. A pesar de que dentro de las estrategias adhesivas disponibles, las técnicas de grabado y lavado en tres pasos, así como la de autograbado en dos pasos son consideradas como estándar de oro ${ }^{(16,17)}$,el grabado y lavado en dos pasos es la técnica más utilizada en nuestro medio, arrojando también resultados satisfactorios ${ }^{(18,19)}$.

\section{Objetivo}

Evaluar y comparar la relación entre la resistencia de unión adhesiva inmediata a esmalte y la microfiltración de dos sistemas restauradores a base de resina compuesta por medio de los ensayos de microtracción y microfiltración.

La hipótesis planteada fue que a mayor valor de resistencia de unión existirá un menor grado de microfiltración.

\section{Materiales y métodos}

\section{Obtención de las muestras:}

La muestra fue constituida por 40 terceros molares con corona sana sin importar el estado de desarrollo radicular. Los mismos fueron obtenidos en el servicio de block quirúrgico de la Facultad de Odontología, teniendo indicación de extracción por motivos ajenos a esta investigación. Los dientes fueron colectados después de la obtención del consentimiento escrito de los pacientes y de la aprobación del proyecto por el comité de ética de la Facultad de Odontología UDELAR (No. De Exp. 215/14). Una vez extraídos, los molares fueron limpiados con curetas periodontales y sometidos a profilaxis con piedra pómez y agua, luego fueron almacenados en Cloramina T 0,5\% por 7 días y posteriormente en agua destilada a una temperatura de $3^{\circ}$ a $5^{\circ} \mathrm{C}$ por no más de 3 meses. Este procedimiento se encuentra protocolizado por el Banco de Dientes Humanos de la Facultad de Odontología (Exp. 091100-000933-11 Consejo de la Facultad de Odontología). 
Todas las restauraciones fueron realizadas en un mismo lote y las muestras para cada prueba fueron distribuidas de manera aleatoria (generado por el sitio web www.randomizer.org) en dos grupos de 20 molares cada uno, dependiendo del sistema restaurador utilizado, en:

Grupo "Z": 20 molares restaurados con Adper Single bond 2+ Filtek Z250 XT(3M ESPE; ST PAUL, MN, USA).

Grupo "T": 20 molares restaurados con Tetric $\mathrm{N}$ Bond + Tetric N Ceram (Ivoclar Vivadent; Schaan, Liechtenstein, Alemania).

La manipulación de todos los materiales fue realizada respetando las indicaciones del fabrican- te. Ambos sistemas adhesivos se aplicaron previo acondicionamiento del esmalte con ácido fosfórico al $37 \%$ por un tiempo de 15 segundos (Dentsply; Petrópolis, RJ, Brasil). Ambos sistemas restauradores fueron seleccionados de igual coloración (A2) a modo de no tener variables de polimerización inducidas por sus pigmentos. Ambas resinas compuestas poseen relleno nanohíbrido por lo que esta variable también estuvo controlada a modo de no producir sesgos. La información referente a la composición, indicaciones del fabricante y lote se encuentran detalladas en la Tabla 1.

Tabla 1. Información de composición, manipulación y lote indicados por el fabricante de los sistemas restauradores (adhesivo + resina compuesta restauradora) utilizados en el presente estudio.

\begin{tabular}{|c|c|c|c|c|}
\hline Nombre comercial & Fabricante & Composición & Modo de uso & LOTE \\
\hline Filtek Z250 XT & 3M ESPE & $\begin{array}{l}\text { Matriz: Bis - GMA, Bis-EMA, } \\
\text { TEGDMA, PEGDMA y UDMA. } \\
\text { Relleno: Nanohíbrido (sílice, } \\
\text { zirconia, cluster de zirconia/síli- } \\
\text { ce). 82\% de carga en peso. } \\
\text { Silano }\end{array}$ & $\begin{array}{l}\text { Los sistemas adhesivos recomendado por el fa- } \\
\text { bricante son: Single Bond de } 3 \mathrm{M}^{\mathrm{TM}} \text { o los Sistemas } \\
\text { Adhesivos Multipropósito Scotchbond } \mathrm{d}^{\mathrm{TM}} \text { de } 3 \mathrm{M}^{\mathrm{TM}} \\
\text { Aplicación de forma incremental } \\
\text { (incrementos de hasta } \\
2,5 \mathrm{~mm} \text { ) } \\
\text { Fotopolimerizar } 20 \mathrm{~s}\end{array}$ & N793196 \\
\hline Tetric N ceram & $\begin{array}{c}\text { Ivoclar } \\
\text { Vivadent }\end{array}$ & $\begin{array}{l}\text { Matriz: Bis - GMA, UDMA, Bis - } \\
\text { EMA, TEGDMA. } \\
\text { Relleno: Nanohíbrido (vidrio } \\
\text { de bario, trifloruro de iterbio, } \\
\text { dióxido de silicio). } 63,5 \% \text { de } \\
\text { carga en peso. } \\
\text { Silano }\end{array}$ & $\begin{array}{l}\text { El Sistema adhesivo recomendado por el fabri- } \\
\text { cante es el Tetric N-bond. } \\
\text { Aplicación de forma incremental } \\
\text { (incrementos de hasta } \\
2 \mathrm{~mm} \text { ) } \\
\text { Fotopolimerizar } 20 \mathrm{~s} \text {. }\end{array}$ & V16057 \\
\hline Adper Single Bond 2 & 3M ESPE & $\begin{array}{l}\text { Matriz: Bis - GMA, HEMA, } \\
\text { dimetacrilatos } \\
\text { Relleno: Nano partícula de sílice } \\
\text { (10\% de carga en peso) } \\
\text { Solvente: etanol y agua }\end{array}$ & $\begin{array}{l}\text { Acondicionamiento con ac. fosfórico al } 37 \% \text { por } \\
15 \mathrm{~s} \text {. } \\
\text { Lavado ( } 10 \mathrm{~s} \text {.) y secado. } \\
\text { Aplicación de adhesivo frotando } 15 \mathrm{~s} \text {, colocar } \\
\text { dos capas. } \\
\text { Secado } 5 \mathrm{~s} \text {. } \\
\text { Fotopolimerizar } 10 \mathrm{~s} \text {. }\end{array}$ & N703454 \\
\hline Tetric N Bond & $\begin{array}{c}\text { Ivoclar } \\
\text { Vivadent }\end{array}$ & $\begin{array}{l}\text { Matriz: Bis - GMA, UDMA, } \\
\text { metacrilato de hidroxietilo. } \\
\text { Relleno: Nano partícula de sílice } \\
\text { (1\% de carga en peso) } \\
\text { Solventes: etanol y agua }\end{array}$ & $\begin{array}{l}\text { Acondicionamiento con ac. fosfórico al 37\% por } \\
\qquad 15-30 \mathrm{~s} \text {. } \\
\text { Lavado }(5 \mathrm{~s} \text {.) y secado. } \\
\text { Aplicación del adhesivo frotando contra las } \\
\text { paredes por } 10 \mathrm{~s} \text {. } \\
\text { Secado. } \\
\text { Fotopolimerizar } 10 \mathrm{~s} \text {. }\end{array}$ & U34550 \\
\hline
\end{tabular}




\section{Ensayo de Microtracción}

La mitad de los dientes asignados a cada uno de los grupos fue utilizada para el ensayo de microtracción $(n=10)$. Las caras vestibulares de los molares fueron desgastadas con lija 600p con agua y en movimientos de " 8 " para obtener una superficie de esmalte plana y lisa. Sobre esta superficie se realizó el tratamiento adhesivo donde inicialmente la superficie fue acondicionada con ácido fosfórico al 37\% por 15 segundos. Pasado ese tiempo la superficie fue lavada enérgicamente con agua a presión por 15 segundos seguidos de un secado con aire a presión en dirección oblicua a la superficie. Posteriormente se aplicó el sistema adhesivo según el grupo correspondiente siguiendo las indicaciones del fabricante y fotopolimerizando la capa de adhesivo con una unidad de fotocurado LED
Optilight Max (Gnatus; Ribeirao Preto, Brasil) previamente analizada con un radiómetro Bluephase Meter (Ivoclar Vivadent; Schaan, Linchenstein, Alemania) en una potencia de $980 \mathrm{mw} / \mathrm{cm}^{2}$.

Luego, se confeccionó sobre la cara vestibular un bloque de resina de $4 \mathrm{~mm}$ de altura y $5 \mathrm{~mm}$ de lado, agregándolo en forma incremental en capas de $2 \mathrm{~mm}$, fotocurando cada incremento según las indicaciones del fabricante. Las piezas fueron almacenadas 24 horas en agua destilada a $37^{\circ} \mathrm{C}$ a modo de dar tiempo a que finalicen las reacciones de polimerización y liberar las tensiones inducidas por la misma. Luego, mediante corte con micrótomo Gellings-Hamco (Hamco Machines INC.; Rochester, NY, USA) se confeccionaron cuerpos de prueba de $1 \mathrm{~mm}^{2}$ de sección (palitos) los cuales eran mitad esmalte y mitad resina compuesta (Fig. 1).

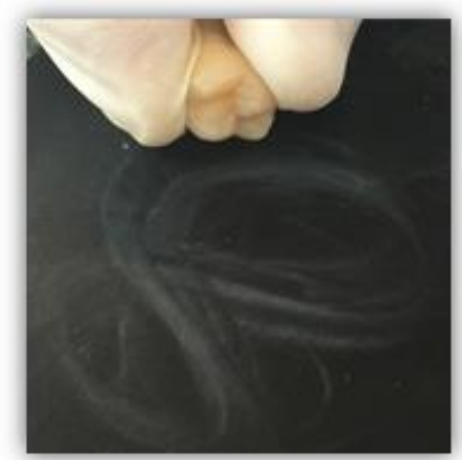

a)

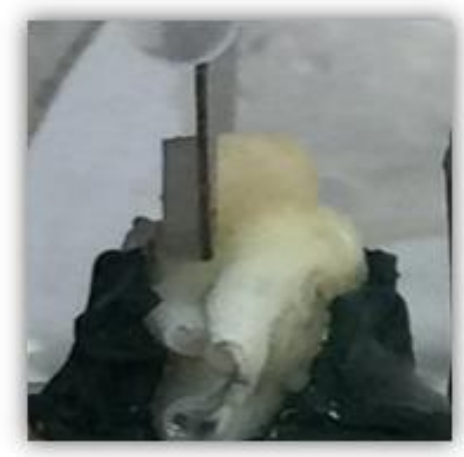

d)

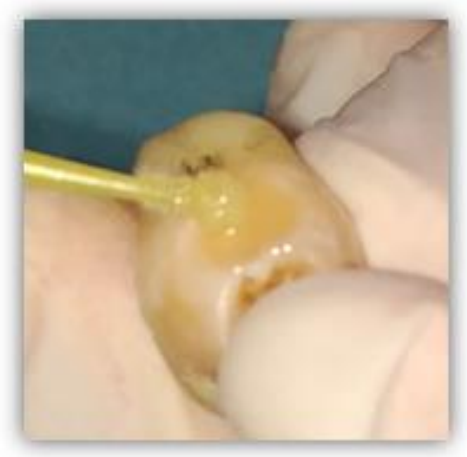

b)

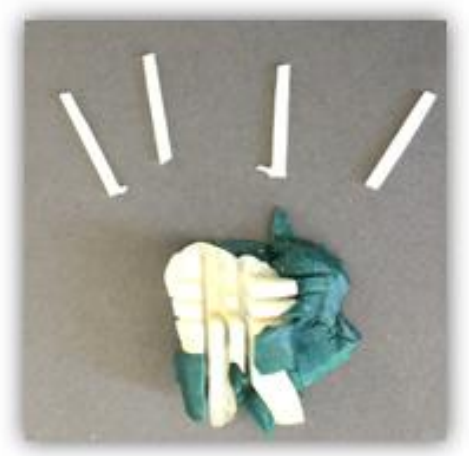

e)

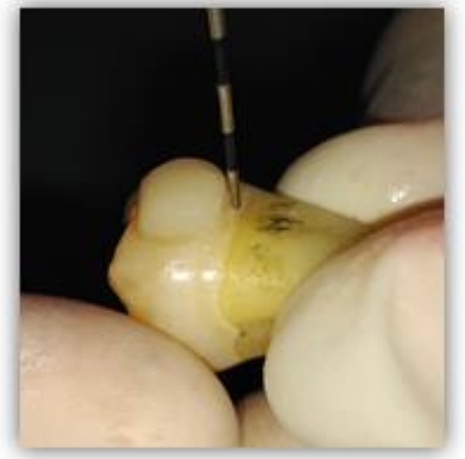

c)

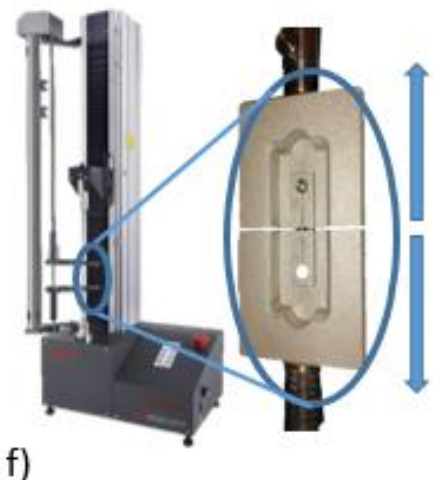

Fig. 1. Representación del proceso de obtención de cuerpos de prueba para ensayo de microtracción a) Obtención de superficie plana y lisa en cara vestibular. b) Realización de la estrategia adhesiva. c) Confección de bloque en resina compuesta. d) Corte mediante micrótomo del bloque de resina. e) Cuerpos de prueba obtenidos. f) Ensayo de microtracción en máquina de ensayos universales. 
El ensayo de microtracción se realizó utilizando una máquina de ensayos universales MTS SANS CMT 2000 5Kn (MTS Systems Corporation; Shanghai China), a una velocidad de $1 \mathrm{~mm} / \mathrm{min}$ y una celda de carga de $100 \mathrm{~N}$.

El cálculo de la resistencia de unión $(\mathrm{MPa})$ se realizó mediante la siguiente formula:

$$
\mu T B S(M P a)=F(N) / A\left(m m^{2}\right)
$$

donde $\mu \mathrm{TBS}$ es la resistencia de unión a microtracción en MPa, F es la fuerza máxima medida por la máquina de ensayos universal en Newton, y A es el área de sección trasversal del cuerpo de prueba calculado por el ancho x profundidad.

Los cuerpos de prueba fueron sometidos a ensayos de microtracción siguiendo la norma ISO $11405^{(14)}$. El modo de falla fue analizado en cada una de las muestras mediante un estereoscopio $(10 \mathrm{x})$ y clasificado en fallas:
a) Adhesivas
b) Cohesivas
c) Mixtas

\section{Ensayo de Microfiltración}

Para el ensayo de microfiltración, fueron utilizados un total de 10 dientes por grupo $(n=10)$. Se realizó una cavidad en esmalte y dentina a nivel del tercio medio de la cara vestibular utilizando instrumental rotatorio de alta velocidad con irrigación Kavo EXTRA torque 505C (Kavo Dental; Schwaben, Alemania) y una fresa cilíndrica de diamante de grano medio 100-110 micron/1.6mm diámetro (Microdont Dental Products; Monsei, NY, USA), obteniendo una cavidad de $3 \mathrm{~mm}$ de diámetro y $1 \mathrm{~mm}$ de profundidad en dentina. La fresa fue cambiada cada 3 cavidades. Las cavidades fueron estandarizadas utilizando una sonda periodontal American Eagle (American Eagle Instruments Inc.; Missoula, Montana, USA) y en caso de no respetarse dichas medidas la pieza fue descartada. Se realizó el tratamiento adhesivo del grupo correspondiente siguiendo las indicaciones del fabricante. Se colocó la resina compuesta agregando capas consecutivas de $2 \mathrm{~mm}$ de espesor hasta obturar toda la cavidad. El adhesivo y resina fueron fotopolimerizadas con una unidad GNATUS Optilight Max. Una vez finalizada la restauración, la misma fue terminada con piedra de diamante grano extrafino 30 micron/0,16 diámetro (Microdont Dental Products; Monsei, NY, USA) y pulida con discos de grano medio y extrafino (Microdont Dental Products; Monsei, NY, USA)hasta obtener una superficie lisa. Las piezas restauradas fueron almacenadas 24 hrs en agua destilada a $37^{\circ} \mathrm{C}$. Finalmente, las muestras fueron selladas con esmalte sintético Colorama transparente (L'Oréal Brasil; San Pablo, Brasil), aplicándole 2 capas en toda la extensión de su raíz y límite amelo-cementario (con excepción de $1 \mathrm{~mm}$ alrededor del borde cavo superficial) a modo de evitar posibles filtraciones radiculares o apicales.

Para el ensayo de microfiltración, las muestras fueron sometidas a 500 ciclos térmicos en termocicladora (Termocycle; Biopdi, San Pablo, Brasil) entre $5^{\circ}$ y $55^{\circ} \mathrm{C}$, siendo cada baño de 20 segundos, con intervalos entre baños de 10 segundos, siguiendo las indicaciones de la normativa ISO/TS $11405^{(14)}$. De acuerdo con algunos estudios, 500 ciclos es suficiente para observar un efecto de microfiltración en la interfaz, y que el aumento en el número de ciclos no está asociado a un aumento del mismo. ${ }^{(20,21)}$ Finalmente, las muestras fueron sumergidas en fucsina básica en solución de propilenglicol al 0,5\% por $24 \mathrm{hrs}$, siguiendo protocolos previamente establecidos, ${ }^{(22,23)}$. Finalmente, las muestras fueron sumergidas en fucsina básica en solución de propilenglicol al $0,5 \%$ por $24 \mathrm{hrs}$, siguiendo protocolos previamente establecidos, $(22,23)$ y se cortaron sagitalmente con un micrótomo Gellings-Hamco (Hamco Machines INC.; Rochester, NY, USA) para ser evaluadas (Fig. 2).”.

Las muestras fueron analizadas mediante un estereoscopio ROSSBACH YZ-6 (Rossbach de México; Ciudad de México, México), y clasificadas de acuerdo al grado de penetración del colorante en:

0 - No hay penetración del colorante

1- Colorante penetró en las paredes del esmalte

2- Colorante penetró en las paredes hasta dentina

3- Colorante llegó hasta el piso de la cavidad 
Todas las observaciones fueron realizadas por el mismo investigador (Ver Fig. 3).

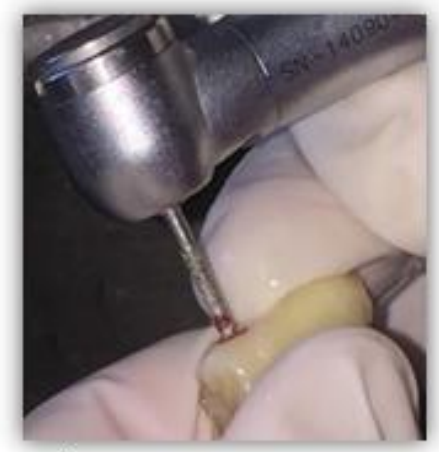

a)

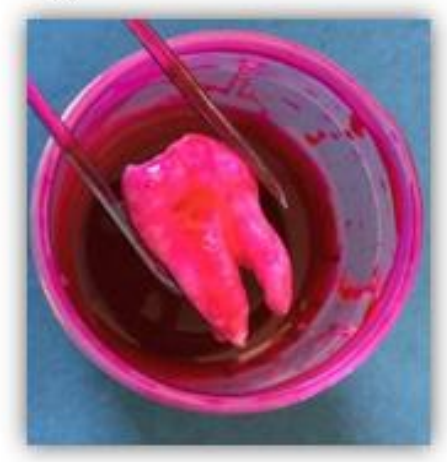

d)

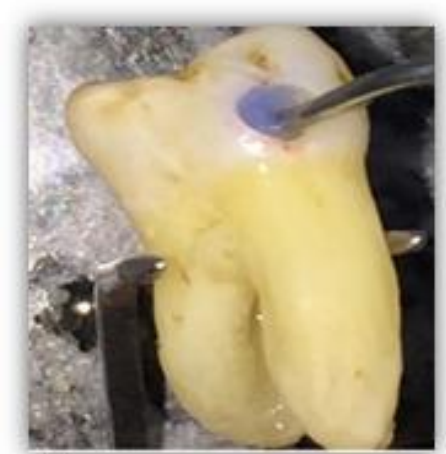

b)

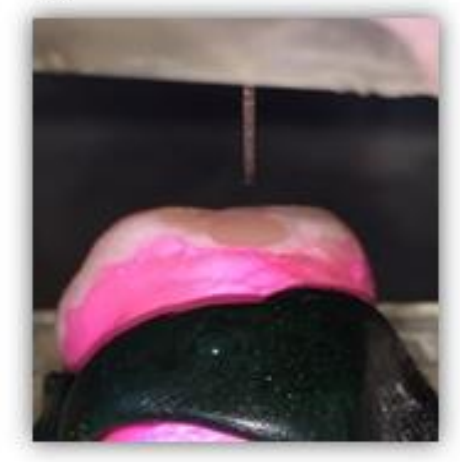

e)

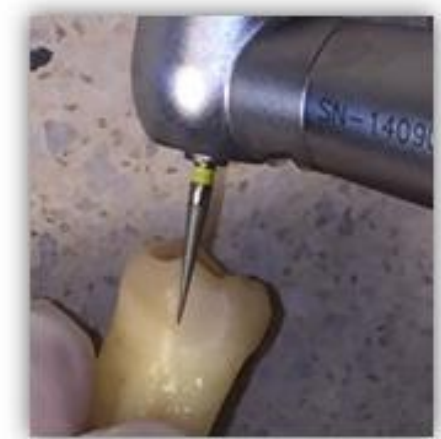

c)

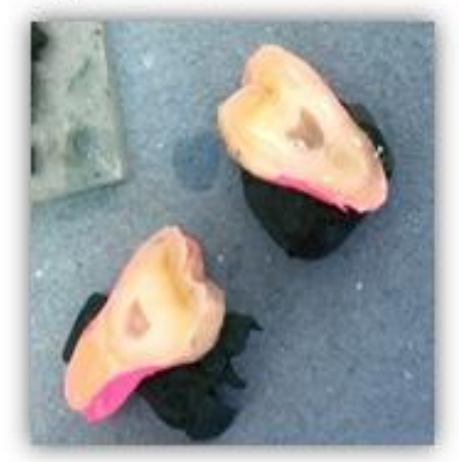

f)

Fig. 2. Representación del proceso de preparación de las muestras para ensayo de microfiltración a) Tallado de cavidades clase 5 en cara vestibular. b) Realización de estrategia adhesiva. c) Obturación con resina compuesta, acabado y pulido de la superficie. d) Inmersión en colorante luego de realizar proceso de termociclado. e) y f) Corte de las muestras a la mitad mediante micrótomo.

\section{Análisis estadístico}

El análisis estadístico fue realizado utilizando el programa Sigma Plot 12.0. Los datos obtenidos de la resistencia de unión en microtracción fueron analizados utilizando la prueba $t$ de Student. Por otro lado, los valores de microfiltración se analizaron utilizando la prueba no paramétrica U Mann-Whitney. Un análisis de correlación de Pearson fue utilizado para determinar el nivel de asociación entre las variables dependientes estudiadas. Para todos los análisis, fue escogido un nivel de significancia de $\alpha=$ 0,05 .

\section{Resultados}

Los valores de resistencia de unión a microtracción son presentados en la Fig. 4. Los resultados del presente estudio mostraron una diferencia estadísticamente significativa entre los grupos ( $\mathrm{p}=0,049$ ), Tetric N Ceram (Ivoclar Vivadent -) presentó mayor valor de resistencia de unión comparado con Filtek Z250XT (3M ESPE ${ }^{\circ}$ ).

Por otro lado, no se encontraron diferencias estadísticamente significativas en el grado de microfiltración $(\mathrm{p}=0,478)$ entre ambos materiales restauradores (Tabla 2). 
a)

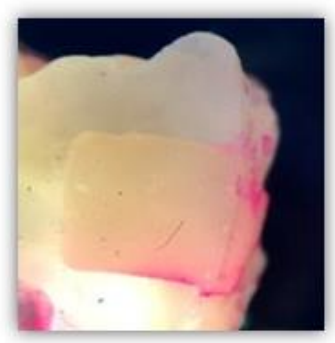

c)

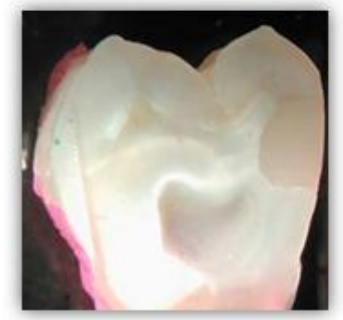

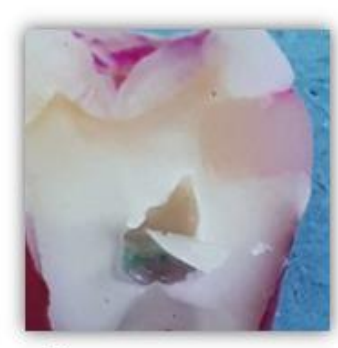

b)

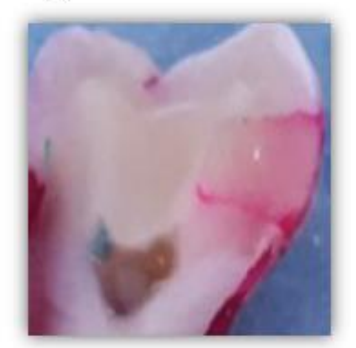

d)
Fig. 3. Clasificación de grado de microfiltración a) Grado 0: no hay penetración del colorante. b) Grado 1: colorante penetró en paredes de esmalte. c) Grado 2: colorante penetró en las paredes hasta dentina. d) Grado 3: Colorante penetró hasta el piso de la cavidad.

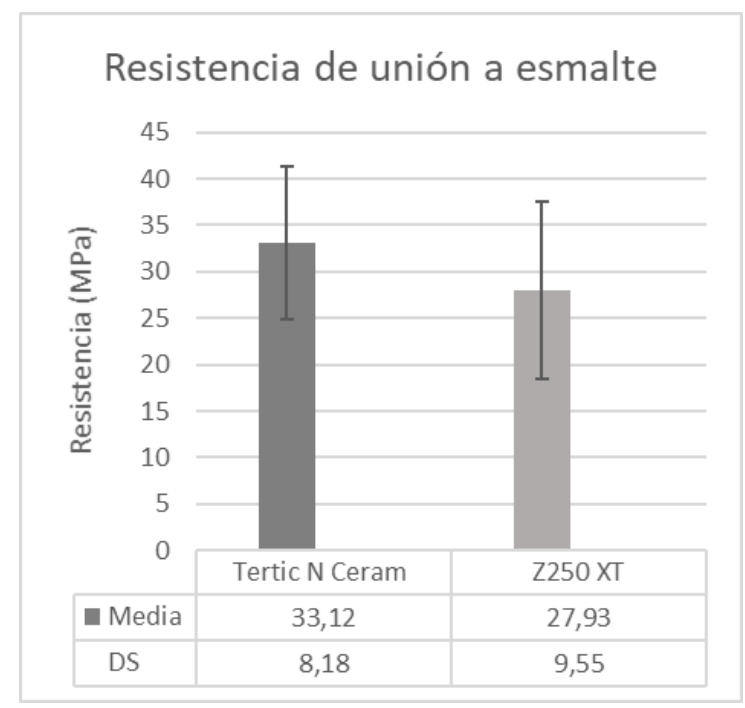

Fig. 4. Valores medios de resistencia de unión y sus respectivas desviaciones estándar (DS) obtenidos luego del ensayo de microtracción.
Tabla 2. Grado de microfiltración de los sistemas restauradores evaluados.

\begin{tabular}{|c|c|c|}
\hline Microfiltración & Tertic N Ceram & Z250 XT \\
\hline GRAD0 0 & 5 & 4 \\
\hline Grado 1 & 2 & 0 \\
\hline Grado 2 & 1 & 3 \\
\hline Grado 3 & 2 & 3 \\
\hline Mediana (RI) & $0.50(0.00-2.25)$ & $2.00(0.00-3.00)$ \\
\hline & $p=0.478$ & \\
\hline
\end{tabular}

El análisis de correlación demostró que no existe una correlación estadísticamente significativa $(p=0,196)$ entre la resistencia de unión a la microtracción y la microfiltración, donde se encontró que el coeficiente de correlación de 0,09 .

\section{Discusión}

Basados en los resultados del presente estudio, no fue posible aceptar nuestra hipótesis, ya que se encontraron diferencias estadísticamente significativas en los valores de resistencia de unión ( $p=0,049)$, no así en el ensayo de microfiltración, presentando ambas resinas un grado de microfiltración similar ( $\mathrm{p}=0,478)$.

Sabemos que el objetivo de la odontología adhesiva es desarrollar materiales que sean capaces de reemplazar la estructura dentaria. La capacidad de sellado (nano-filtración y microfiltración) y resistencia de unión adhesiva son dos de los tipos principales de pruebas de laboratorio utilizados para evaluar el rendimiento de los sistemas de restauración ${ }^{(3)}$.

Para microtracción, los datos obtenidos en el presente estudio corresponden con los de la literatura, siendo de entre 28,57 y $29 \mathrm{MPa}$ para Tetric N Bond, ${ }^{(24,25)}$ y de 24,8 a 29,7 MPa para Adper Single Bond $2^{(26,27)}$.

Es importante mencionar que el presente estudio permitió corroborar que no se ha encontrado una relación directa entre resistencia de unión y microfiltración, al igual que otros trabajos presentes en la literatura ${ }^{(28-31)}$. 
De igual forma, es pertinente destacar que la intención de la prueba de microfiltración no es determinar la sobrevida del material, ya que existe evidencia en la literatura que demuestra que los test de penetración para evaluar la microfiltración no se correlacionan con los parámetros de éxito clínico de la restauración (sensibilidad postoperatoria, retención, coloración marginal) ${ }^{(32)}$. El objetivo del termociclado es simular un envejecimiento del material mientras que la prueba de microfiltración pretende analizar el modo de falla de la interfaz adhesi$\mathrm{va}^{(33)}$. Es por esto que, aun se siguen llevando a cabo trabajos de investigación que intentan predecir el comportamiento de los sistemas de restauración poliméricos utilizando este tipo de ensayo ${ }^{(28-31)}$.

Habiendo discutido los resultados y observando otros trabajos científicos, podemos concluir que la resistencia de unión no es el único factor determinante para comprobar el desempeño de un sistema adhesivo. Además, se debe tener en cuenta otras múltiples variables inherentes al material (tipo y porcentaje relleno, grado de contracción volumétrica, estabilidad hidrolítica, coeficiente de expansión térmica, etc.) ${ }^{(34,35)}$, a la técnica (técnica estratificada, monocapa, factor C, tiempos e intensidad de fotopolimerización, etc.), ${ }^{(35,36)}$ al sustrato (profundidad, tejidos involucrados, tipo de dentición, remoción del barrillo, etc. $)^{(37)}$, y al medio en que estos se encuentran (variabilidad de $\mathrm{pH}$, cargas mecánicas recibidas, etc. $)^{(36,38-41)}$.

Actualmente, podemos decir que los sistemas restauradores cumplen satisfactoriamente con los requisitos establecidos en las normas, logrando un buen desempeño clínico. Es por esto que la selección de un sistema restaurador se ha vuelto un problema menor hoy en día ${ }^{(2,9)}$. Por otro lado, es probable que el "factor operador", que rara vez se estudia, juegue un papel principal en todo el proceso de longevidad ${ }^{(42-44)}$.

Por lo anteriormente mencionado, se hace hincapié en que el profesional conozca el material y su correcta manipulación (respetando los pro- tocolos establecidos) para poder generar una adecuada unión entre el mismo y la estructura dentaria perdurable en el tiempo.

Más estudios que tomen en cuenta estas variables deben ser realizados a modo de poder apoyar nuestras conclusiones y afirmar el rol de otros factores en el desempeño de los sistemas adhesivos, como ensayos clínicos a largo plazo, que sean capaces de registrar con mayor acercamiento las condiciones reales.

\section{Conclusiones}

Los sistemas restauradores utilizados fueron incapaces de prevenir la microfiltración, a pesar de que Tetric N Ceram obtuvo mayores valores de resistencia de unión.

No se encontró una relación entre los valores de resistencia de unión y el grado de microfiltración. La resistencia de unión no es el único factor determinante para predecir el desempeño de un sistema adhesivo, por lo que se deben tener en cuenta otras múltiples variables inherentes al material, al sustrato, a la técnica, y al ambiente en que estos se encuentran.

\section{Agradecimientos}

El presente trabajo fue financiado por el Programa de Apoyo para la Investigación Estudiantil (PAIE) de la Comisión Sectorial de Investigación Científica (CSIC).

\section{Conflicto Interés}

Los autores declaran no tener conflicto de interés respecto a las empresas mencionadas en este trabajo. 


\section{Contribución de autoría}

1. Concepción y diseño del estudio

2. Adquisición de datos

3. Análisis de datos

4. Discusión de los resultados

5. Redacción del manuscrito

6. Aprobación de la versión final del manuscrito

MELC ha contribuido en 1, 2, 3, 4, 5 . MMG ha contribuido en 1, 2, 3, 4, 5 . CECS ha contribuido en 3, 4, 6 .

FMG ha contribuido en 1, 4, 6 .

GSGP ha contribuido en 1, 3, 4, 5, 6 .

\section{Referencias}

1. Correa MB, Peres MA, Peres KG, Horta BL, Barros AD, Demarco FF. Amalgam or composite resin? Factors influencing the choice of restorative material. J Dent. 2012;

2. Demarco FF, Corrêa MB, Cenci MS, Moraes RR, Opdam NJM. Longevity of posterior composite restorations: not only a matter of materials. Dent Mater. 2012; 28 (1): 87-101.

3. Chang C-H, Fang C-L, Hsu J-T, Chen. ChangPen. Cavity Dimension Effect on MOD Dental Restoration Filled with Resin Composite - A Finite Element Interface Stress Evaluation. J Med Biol Eng. 2004; 24 (4).

4. Ishikiriama SK, De Oliveira GU, Maenosono RM, Wang L, Duarte MAH, Mondelli RFL. Wear and surface roughness of silorane composites after $\mathrm{pH}$ cycling and toothbrushing abrasion. Am J Dent. 2014; 27 (4): 195-8.

5. Mjör IA. Problems and benefits associated with restorative materials: side-effects and long-term cost. Adv Dent Res. 1992; 6 (1): 7-16.

6. Mjör IA, Jokstad A. Five-year study of Class II restorations in permanent teeth using amalgam, glass polyalkenoate (ionomer) cerment and resin-based composite materials. J Dent. 1993;21 (6): 338-43.
7. Mjör IA. The reasons for replacement and the age of failed restorations in general dental practice. Acta Odontol Scand. 1997;55 (1): 58-63.

8. Braga SRM, Vasconcelos BT, Macedo MR de P, Martins VRG, Sobral MAP. Reasons for placement and replacement of direct restorative materials in Brazil. Quintessence Int. 2007; 38 (4): e189-94.

9. Opdam NJ, van de Sande FH, Bronkhorst E, Cenci MS, Bottenberg P, Pallesen U, Gaengler P, Lindberg A, Huysmans MC, van Dijken JW. Longevity of Posterior Composite Restorations. J Dent Res. 2014; 93 (10): 943-9.

10. Matos AB, Trevelin LT, Silva BTF da, Francisconi-dos-rios LF, Siriani LK, CArdoso MV. Bonding efficiency and durability: current possibilities. Braz Oral Res. 2017; 31(suppl 1).

11. Hahnel S, Henrich A, Bürgers R, Handel G, Rosentritt M. Investigation of mechanical properties of modern dental composites after artificial aging for one year. Oper Dent. 2010; 35 (4): 412-9.

12. Amano S, Yamamoto A, Tsubota K, Rikuta A, Miyazaki M, Platt JA, Moore BK. Effect of Thermal Cycling on Enamel Bond Strength of Single-step Self-etch Systems. Oper Dent. 2006; 31(5): 616-22.

13. Rossomando KJ, Wendt SL. Thermocycling and dwell times in microleakage evaluation for bonded restorations. Dent Mater. 1995;11 (1): 47-51.

14. ISO/TS 11405:2015(en), Dentistry — Testing of adhesion to tooth structure.

15. Van Meerbeek B, De Munck J, Yoshida Y, Inoue S, Vargas M, Vijay P, Van Landuyt K, Lambrechts P, Vanherle G. Adhesion to enamel and dentin: current status and future challenges. Oper Dent. 2003;

16. Mahn E, Rousson V, Heintze S. Meta-Analysis of the Influence of Bonding Parameters on the Clinical Outcome of Tooth-colored Cervical Restorations. J Adhes Dent. 2015;17 (5): 391403.

17. van Dijken JW V, Pallesen U. Long-term dentin retention of etch-and-rinse and self-etch adhesives and a resin-modified glass ionomer cement in non-carious cervical lesions. Dent Mater. 2008;24 (7): 915-22. 
18. Perdigão J. New Developments in Dental Adhesion. Dent Clin North Am. 2007;51 (2): 333-57.

19. Cardoso MV, de Almeida Neves A, Mine A, Coutinho E, Van Landuyt K, De Munck J, Van Meerbeek B.Current aspects on bonding effectiveness and stability in adhesive dentistry. Aust Dent J. 2011;56 Suppl 1:31-44.

20. Wahab FK, Shaini FJ, Morgano SM. The effect of thermocycling on microleakage of several commercially available composite Class V restorations in vitro. J Prosthet Dent [Internet]. 2003; 90 (2): 168-74. Available from: http:// www.ncbi.nlm.nih.gov/pubmed/12886210

21. Pazinatto FB, Campos BB, Costa LC, Atta MT. Effect of the number of thermocycles on microleakage of resin composite restorations. Pesqui Odontol Bras. 2003; 17 (4): 337-41.

22. Güçlü ZA, Dönmez N, Hurt AP, Coleman NJ. Characterisation and microleakage of a new hydrophilic fissure sealant-ultraseal XT ${ }^{\oplus}$ hydro $^{\mathrm{TM}}$. J Appl Oral Sci. 2016;24 (4): 344-51.

23. Okida RC, Martins TM, Briso ALF. In vitro evaluation of marginal leakage in bonded restorations, with mechanical or chemical-mechanical (Carisolv) removal of carious tissue. Braz Oral Res. 2007; 21 (2): 176-81.

24. Bedran-de-Castro AKB, Pereira PNR, Pimenta LAF, Thompson JY. Effect of thermal and mechanical load cycling on microtensile bond strength of a total-etch adhesive system. Oper Dent. 29 (2): 150-6.

25. Shadman N, Farzin Ebrahimi S, Abrishami A, Sattari H. Shear bond strength of three adhesive systems to enamel and dentin of permanent teeth. J Dent Med. 2012; 25 (3): 202-10.

26. Ivoclar-Vivadent. Tetric ${ }^{\odot} \mathrm{N}$-Collection Un completo sistema restaurativo nano-optimizado.

27. De Nordenflycht D, Kaplan M, Montecinos V, Báez A. Resistencia microtraccional de capa de adhesivo contaminada con sangre. Rev Clínica Periodoncia, Implantol y Rehabil Oral. 2013; 6 (3): 118-22.

28. Ateyah NZ, Elhejazi AA. Shear bond strengths and microleakage of four types of dentin adhesive materials. J Contemp Dent Pract. 2004; 5 (1): 63-73.
29. Fortin D, Swift EJ, Denehy GE, Reinhardt JW. Bond strength and microleakage of current dentin adhesives. Dent Mater. 1994;10 (4): 253-8.

30. Abdelrahman M, Abdelrahman $\mathrm{MH}$, Mahmoud EM, Ghoneim MM, Kammar AA. Comparative study of microleakage and shear bond strength between bulk fill and self adhesive flowable composite resins. Alexandria Dent J. 2016; 41 (3): 322-7.

31. Chowdary Mandava R, Gurunathan D, Ramakrishnan M, Subramanian E. Comparison of Microleakage and Shear Bond Strength (SBS) of Dyad Flow and Tetric Flow in Primary Molars: An In-vitro Study. J Clin Diagnostic Res. 2018; 12 (10).

32. Heintze SD. Clinical relevance of tests on bond strength, microleakage and marginal adaptation. Dent Mater. 2013; 29 (1): 59-84.

33. Gale MS, Darvell BW. Thermal cycling procedures for laboratory testing of dental restorations. J Dent. 1999; 27 (2): 89-99.

34. Ilie N, Hilton TJ, Heintze SD, Hickel R, Watts DC, Silikas N, Stansbury JW, Cadenaro M, Ferracane JL.Academy of Dental Materials guidance-Resin composites: Part I-Mechanical properties. Dent Mater. 2017; 33 (8): 880-94.

35. Nedeljkovic I, Teughels W, De Munck J, Van Meerbeek B, Van Landuyt KL. Is secondary caries with composites a material-based problem? Dent Mater. 2015; 31 (11):e247-77.

36. Demarco FF, Collares K, Correa MB, Cenci MS, Moraes RR de, Opdam NJ. Should my composite restorations last forever? Why are they failing? Braz Oral Res. 2017;31 (suppl 1).

37. van de Sande FH, Opdam NJ, Rodolpho PADR, Correa MB, Demarco FF, Cenci MS. Patient risk factors' influence on survival of posterior composites. J Dent Res. 2013; 92 (7 Suppl): 78S-83S.

38. Hamburger JT, Opdam NJM, Bronkhorst EM, Huysmans MCDNJM. Indirect restorations for severe tooth wear: fracture risk and layer thickness. J Dent. 2014; 42 (4): 413-8.

39. Leloup G, D'Hoore W, Bouter D, Degrange M, Vreven J. Meta-analytical review of factors involved in dentin adherence. J Dent Res. 2001; 80 (7): 1605-14.

40. De Munck J, Mine A, Poitevin A, Van Ende A, Cardoso MV, Van Landuyt KL, Peumans M, Van Meerbeek B. Meta-analytical Review of Pa- 
rameters Involved in Dentin Bonding. J Dent Res. 2012; 91 (4): 351-7.

41. Van Meerbeek B, Peumans M, Poitevin A, Mine A, Van Ende A, Neves A, De Munck J. Relationship between bond-strength tests and clinical outcomes. Dent Mater. 2010; 26 (2): e100-21.

42. Laske M, Opdam NJM, Bronkhorst EM, Braspenning JCC, Huysmans MCDNJM. Longevity of direct restorations in Dutch dental practices. Descriptive study out of a practice based research network. J Dent. 2016; 46: 12-7.

43. Heaven TJ, Gordan VV, Litaker MS, Fellows JL, Brad Rindal D, Firestone AR, Gilbert
GH; National Dental PBRN Collaborative Group. Agreement among dentists' restorative treatment planning thresholds for primary occlusal caries, primary proximal caries, and existing restorations: Findings from The National Dental Practice-Based Research Network. J Dent. 2013;41 (8): 718-25.

44. Lucarotti PSK, Holder RL, Burke FJT. Outcome of direct restorations placed within the general dental services in England and Wales (Part $1)$ : variation by type of restoration and re-intervention. J Dent. 2005;33 (10): 805-15.

Matías Mederos: matiasmederos@odon.edu.uy 